\title{
Characteristic Analysis of VolP Traffic for Wireless Networks In Comparison with CBR using QualNet Network Simulator
}

\author{
Raghavendra Ganiga \\ Department of Information and \\ Communication Technology, \\ Manipal Institute of Technology, \\ Manipal University, Manipal, \\ India.
}

\author{
Balachandra Muniyal \\ Department of Information and \\ Communication Technology, \\ Manipal Institute of Technology, \\ Manipal University, Manipal, \\ India.
}

\author{
Pradeep \\ Software Engineering Branch, \\ Dept. of ISE, PESIT, \\ Visvesvaraya Technology \\ University, Karnataka, India
}

\begin{abstract}
Voice over Internet Protocol (VoIP) is one of the most important technologies in the world of communication. Around 20 years of research on VoIP, some Quality of Service $(\mathrm{QoS})$ problems of VoIP are still remaining. During the past decade and with growing of wireless technologies, we have seen that many papers turn their concentration from Wired-LAN to Wireless-LAN. VoIP over Wireless LAN (WLAN) faces many challenges, due to the loose nature of wireless network. Issues like providing QoS at a good level, dedicating capacity for calls and having secure calls is more difficult rather than wired LAN. Therefore VoIP over WLAN (VoWLAN) remains a challenging research topic. Popularity of Voice over IP (VoIP) application such as Google Talk, Skype and MSN Messenger is making VoIP over WiMAX an eye-catching market and a driving force for both carriers and equipment suppliers. In this paper experimentally measured VoIP traffic and CBR traffic over the WiMAX network by using the different routing algorithm using QualNet 4.5.1 network simulation tool. In addition, this paper also shows that VoIP application can be best served with WiMAX rather than the CBR. Future work will involve simulating scenarios that are closer to the real world models.
\end{abstract}

\section{General Terms}

Voice over Internet Protocol, Routing Protocol, QualNet Simulator.

\section{Keywords}

VoIP, CBR, QoS, QualNet 4.5.1, WiMAX.

\section{INTRODUCTION}

The ability to communicate properly over long distances has become an integral part of society today. Businesses are expanding to different regions in the world, but need to keep the same deadlines. This means it is necessary for employees in two different regions to communicate with each other over long distances, cheaply and trouble free. The public switched telephone network (PSTN) [6] has developed itself to accommodate these requirements. The internet has become a very popular means of communication in a very short period of time. It was set up as a network where people could share files and access other peoples work. It has since established itself as a massive communication infrastructure that provides many services such as electronic mail .In the recent years it has further developed itself into providing Internet Telephony or Voice over internet protocol (VOIP).

The IEEE 802.16 standard (popularly known as WiMAX) defines high range and high bandwidth wireless access for fixed and mobile users. WiMAX networks provide voice, data and video services. The fixed wireless version of WiMAX has the potential to replace DSL and/or cable internet for broadband service. The mobile wireless version of WiMAX [7] has the potential to replace, or at least enhance, cellular networks, including $3 \mathrm{G}$ networks. In this paper the performance of different routing protocols for VoIP application in WiMAX networks are evaluated.

The outline of the rest of the paper is as follows: In Section 2 we give a general overview of the VoIP and IEEE 802.16 WiMax network; In Section 3 Describes the routing protocols used with VoIP and CBR connection. Section 4 we analyses the characteristic of VoIP [1] traffic for wireless network In comparison with CBR using network simulator. In Sections 5 we describe our experiments and show our results; Section 6 concludes the paper.

\section{BACKGROUND}

In this section we will give general overview of the VoIP Architecture and IEEE 802.16 Wi-Max network.

\subsection{Overview of VoIP}

VoIP (Voice over IP) is an IP telephony term for a set of facilities used to manage the delivery of voice information over the Internet. VoIP involves sending voice information in digital form in discrete packets rather than by using the traditional circuit-committed protocols of the Public Switched Telephone Network (PSTN). A major advantage of VoIP and Internet telephony is that it avoids the tolls charged by ordinary telephone service by making use of the existing DSL/Cable lines, thereby reducing the overall call cost to the service provider and the end customer [2].

The QualNet VoIP traffic generator model simulates end to end voice conversations. The initiator and receiver generate real time traffic with an exponential distribution function that simulates a real life telephone conversation. VoIP uses a wide 
variety of protocols to provide the above mentioned services. H323 and SIP are the broadly used protocols for call signaling and management purposes. Once the session is established, the actual data is carried over RTP packets. RTCP is a companion control protocol to RTP that is used to collect endto-end information about the quality of the session to each participant.

VoIP systems employ session control protocols to control the set-up and tear-down of calls as well as audio codecs which encode speech allowing transmission over an IP network as digital audio via an audio stream. Codec use is varied between different implementations of VoIP (and often a range of codecs are used); some implementations rely on narrowband and compressed speech, while others support high fidelity stereo codecs.

VoIP [5] represents the next generation in communication services. By moving voice services to the data network, we eliminate a separate, managed voice infrastructure and dramatically reduce the cost of telephone moves, add and changes. Placing voice calls over the data network will require Stanford to take steps, including equipment upgrades, to support time sensitive applications such as voice.

The advantages of selecting IP telephony include providing for accommodation of long range developments in networking and telephony services and products, potentially lowering certain personnel and infrastructure costs over time. By controlling the network that VoIP calls travel on, you control the "quality" of the call, allowing businesses to have near "toll quality" calling as we all have been used to for the last 100 years.

By using VOIP telephone calls over the Internet do not incur a surcharge beyond what the user is paying for Internet access, much in the same way that the user doesn't pay for sending individual e-mails over the Internet. Digital lines increase the quality of your calls. Save money on your international calls with VoIP's low rates to countries all around the world.

VoIP can turn a standard Internet connection into a way to place free phone calls. The practical upshot of this is that by using some of the free VoIP software that is available to make Internet phone calls, you're bypassing the phone company entirely. VoIP is a revolutionary technology that has the potential to completely rework the world's phone system. VoIP is basically a clever "reinvention of the wheel." It's an emerging technology, which will more than likely one day replace the traditional Phone system entirely.

\subsection{Overview of IEEE 802.16 and IEEE 802.16e Network}

The IEEE 802.16 standard, including MAC layer and PHY layer specifications, defines the air interface and associated functions of the broadband wireless access system supporting multimedia services. It is designed for high-range and high- bandwidth wireless access, or Wireless Metropolitan Area Network (Wireless MAN). The bandwidth is up to $70 \mathrm{Mbps}$ and radio range can go up to 50 kilometres (31 miles). Its major advantages includes high bandwidth and large coverage range, Multiple service with different QoS guarantees, Builtin security, Cost-effective and fast-to-deploy first mile access to public networking, A cost effective alternative that replaces $\mathrm{Wi}-\mathrm{Fi}$ and $3 \mathrm{G} / 4 \mathrm{G}$.

The IEEE 802.16 [1] is also known as WiMAX, which is a certification mark for products that pass conformity and interoperability tests for IEEE 802.16 standards. The basic components of an 802.16 network are Base Stations (BS) and Subscriber Stations (SS) (or Mobile Stations (MS) in 802.16e). The BS connects to the public networks and serves their registered subscriber stations. The SS typically serve a building (commercial or residential, or Wi-Fi hot spots). Both BS and SS are assumed to be static in an 802.16 network (mobility support is added in 802.16e standard). The basic operation mode of an 802.16 network is called Point to MultiPoint (PMP) [11] where SS is only one-hop away from BS and can only communicate with its BS, not other neighbouring SS. An optional operational mode called Mesh mode, has no clear distinction between SS and BS. Stations can talk directly to each other and be more than one-hop away from the BS, and the BS is defined as the station that provides access to the public network, such as the Internet. IEEE 802.16 e added mobility support to IEEE 802.16. [4] It can support mixed fixed and mobile broadcast wireless access networks. In the 802.16e specification, Subscriber Stations (SS) are also referred to as Mobile Stations (MS). Under 802.16e, the MS can handover from one BS to another BS.

\section{ROUTING PROTOCOLS IN QualNet 4.5.1 SIMULATOR}

\subsection{Bellman-Ford Routing Protocol}

Bellman-Ford Routing Algorithm, also known as FordFulkerson Algorithm, is used as a distance vector routing protocol. Routers that use this algorithm have to maintain the distance tables, which tell the distances and shortest path to sending packets to each node in the network. The information in the distance table is always updated by exchanging information with the neighbouring nodes. The number of data in the table equals to that of all nodes in networks. [11] The columns of table represent the directly attached neighbours whereas the rows represent all destinations in the network. Each data contains the path for sending packets to each destination in the network and distance/or time to transmit on that path. The measurements in this algorithm are the number of hops, latency, and the number of outgoing packets.

\subsection{Ad- Hoc on Demand Distance Vector Routing Protocol}

AODV protocol is specially used for mobile ad hoc networks. It provides a quick adaptation to dynamic link condition, link fault, low processing and memory usage overhead. It enables dynamic, self-starting, multihop routing between participating mobile nodes wishing to establish and maintain an ad hoc network. AODV [8] allows mobile nodes to obtain routes quickly for new destinations, and does not require nodes to maintain routes to destinations that are not in active communication. AODV allows mobile nodes to respond to 
link breakages and changes in network topology in a timely manner. It uses sequence numbers to prevent routing loops.

\subsection{Fisheye Routing Protocol}

Fisheye State Routing (FSR)[9] is a link state type protocol that maintains a topology map at each node. FSR differs from the standard link state algorithm in the following reason having only neighbouring nodes exchange the link state information and utilizing only time-triggered, not eventtriggered link state exchanges.

In FSR, every update message doesn't contain information about all nodes in the network. Instead, information about closer nodes is exchanged more frequently than it is done about farther nodes, thus reducing the update message size. The center node has most up to date information about all nodes in the inner circle and the accuracy of information decreases as the distance from node increases. This procedure of dividing the network into different scope levels is done at each node, meaning that it is independent on a central entity. Even if a node doesn't have accurate information about far away nodes, the packets will be routed correctly because the route information becomes more and more accurate as the packet gets closer to the destination. This means that FSR scales well to large networks as the overhead is controlled.

\section{TESTBED DEPLOYMENT}

This section describes the experimental testbed that has been set up to evaluate VoIP traffic and CBR traffic over WiMAX network using QualNet network simulator. Wi-MAX network testbed has been developed using QualNet 4.5.1 simulator [11]. QualNet 4.5.1 has several core components as well as add-on-components. It is state of art simulator for large, heterogeneous networks and the distributed applications that execute on that. Four wireless subnets are created with 1 BS in each subnet shown in figure 1. The BSs from node 1 to node 4 are deployed along a highway to cover the route that node 5 drives from one point home to another point office. [10] The BSs are connected to a switch center node 6 by wired links. When node 5 drives along the highway, node 5 communicates with node 7 and the communication is handovered from BS 1 to BS 2 to BS 3, and finally to BS 4. First we simulated below scenario by sending packet using CBR traffic generator protocol and AODV routing protocol and simulated same scenario by changing the routing protocol. To check the performance of the routing protocol with traffic generator we used VoIP traffic [12] generator protocol and analyzed the result by enabling the different routing protocol

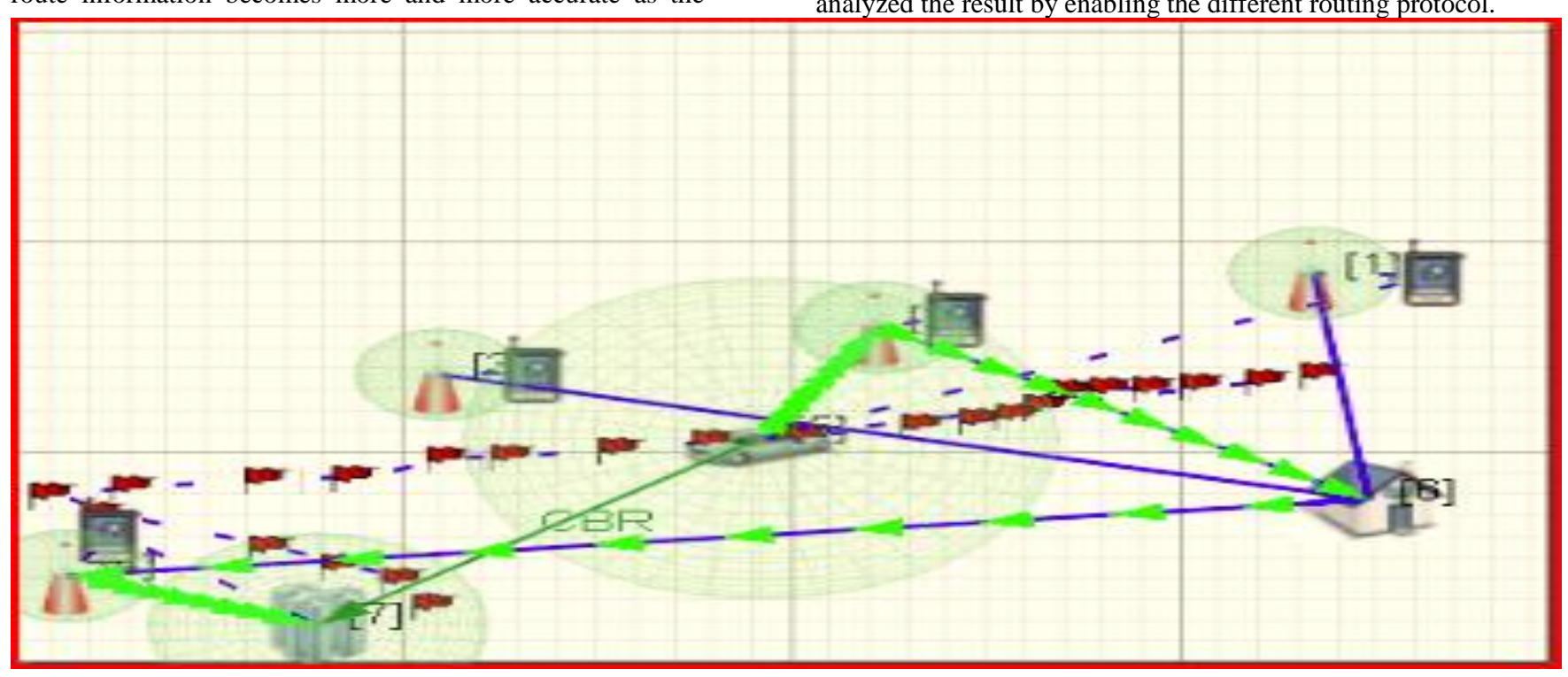

Figure 1: Simulation Scenario

Table 1: Experimental Result

\begin{tabular}{|c|c|c|c|c|}
\hline \multirow{2}{*}{$\begin{array}{c}\text { Routing } \\
\text { Protocol }\end{array}$} & \multicolumn{2}{|c|}{ CBR } & \multicolumn{2}{c|}{ VOIP } \\
\cline { 2 - 5 } & Packets Sent & Packets Received & Packets Sent & 329 \\
\hline AODV & 9400 & 8000 & 376 & 373 \\
\hline Bellman Ford & 9400 & 7342 & 439 & 337 \\
\hline Fisheye & 9400 & 3136 & 437 & \\
\hline
\end{tabular}


Table 2: Average Packet Loss rate in the experiments

\begin{tabular}{|c|c|c|}
\hline Routing Protocol & Packet Loss Rate In CBR & Packet Loss Rate in VoIP \\
\hline AODV & $15.45 \%$ & $12.80 \%$ \\
\hline Bellman Ford & $22.10 \%$ & $16.60 \%$ \\
\hline Fisheye & $67.36 \%$ & $22.86 \%$ \\
\hline
\end{tabular}

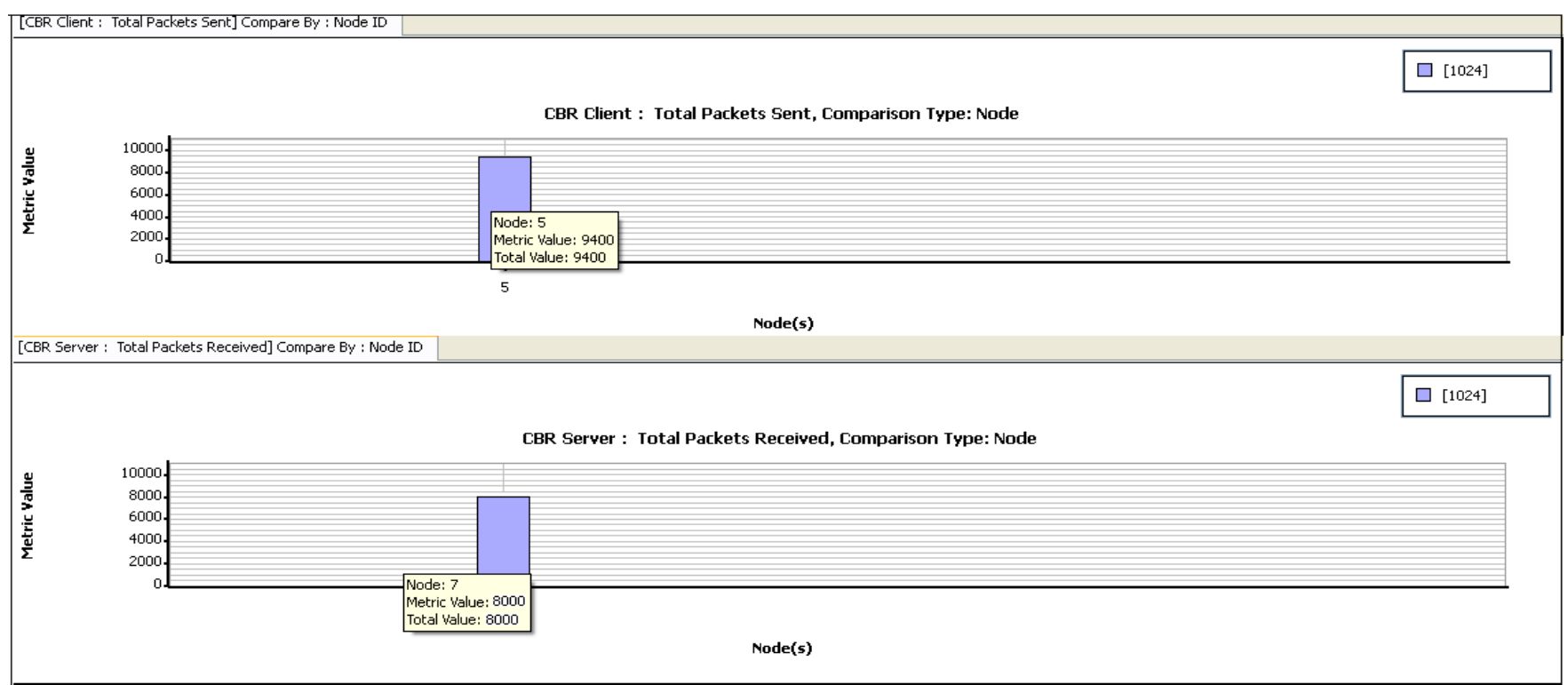

Figure: 2 simulated results for sending high number of packet from CBR Client to CBR Server using AODV routing protocol

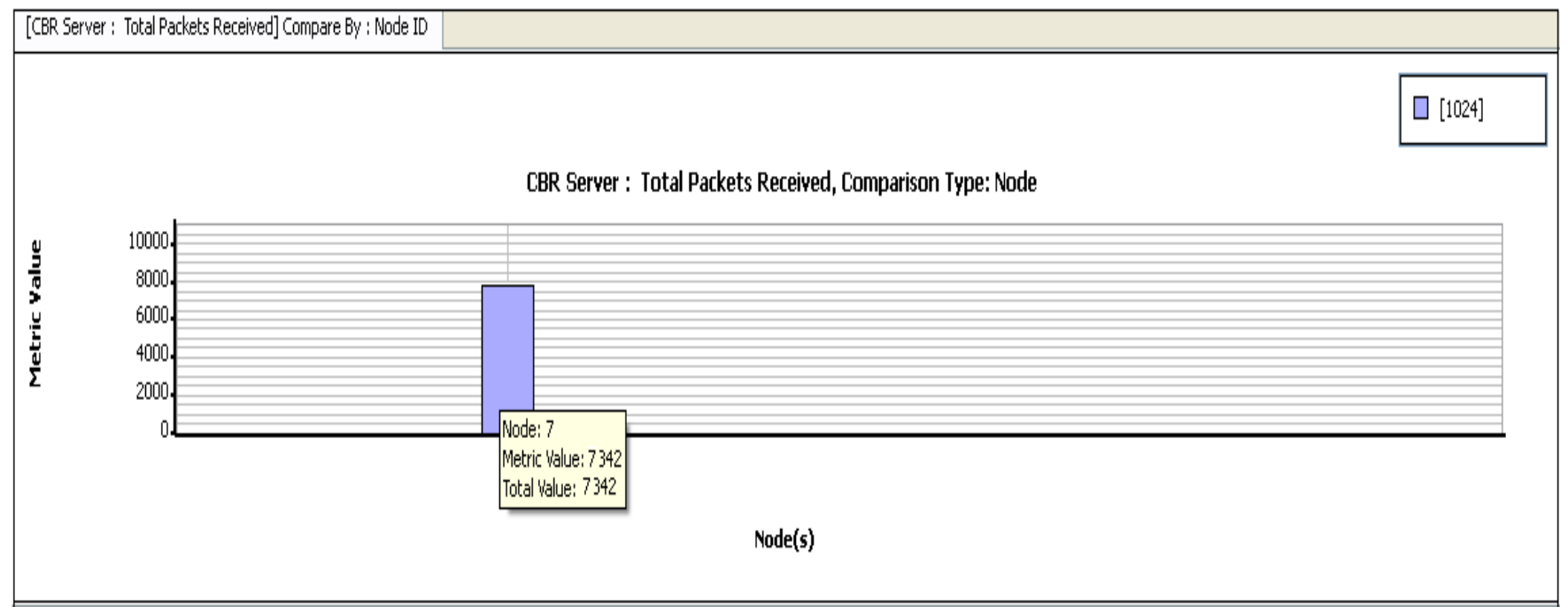

Figure: 3 simulated results for total packet received from CBR Server using Bellman ford routing protocol 


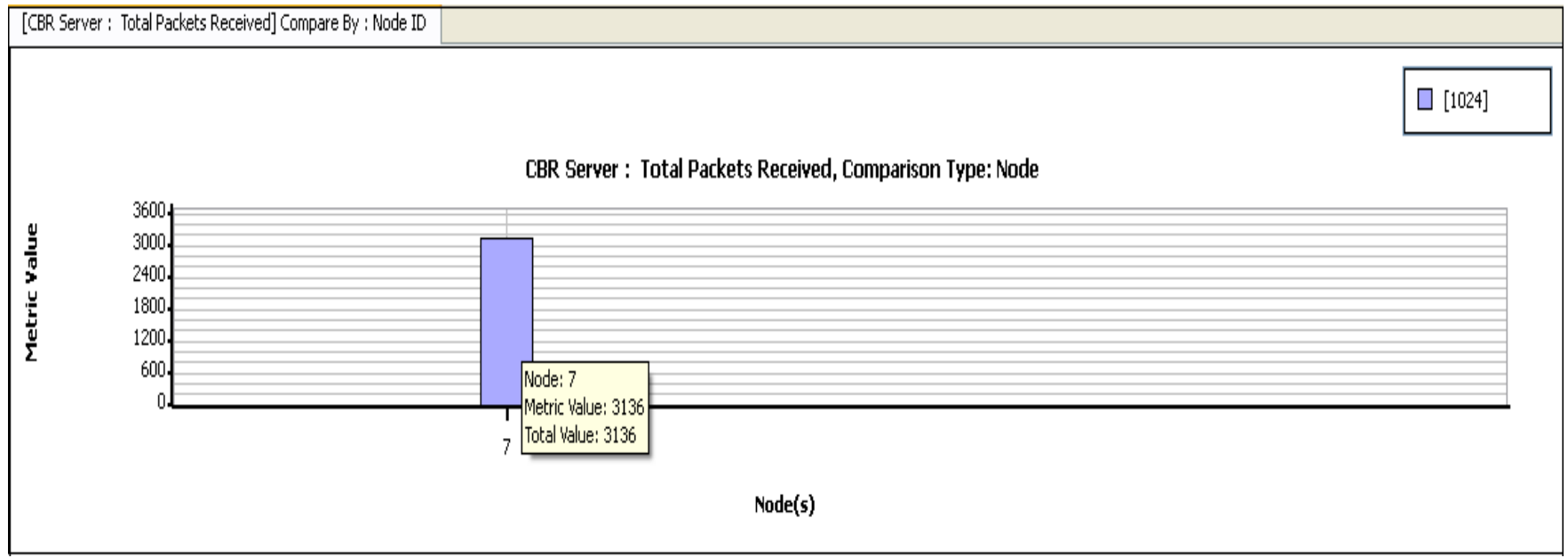

Figure: 4 simulated results for total number of packets received from CBR Server using Fisheye routing protocol

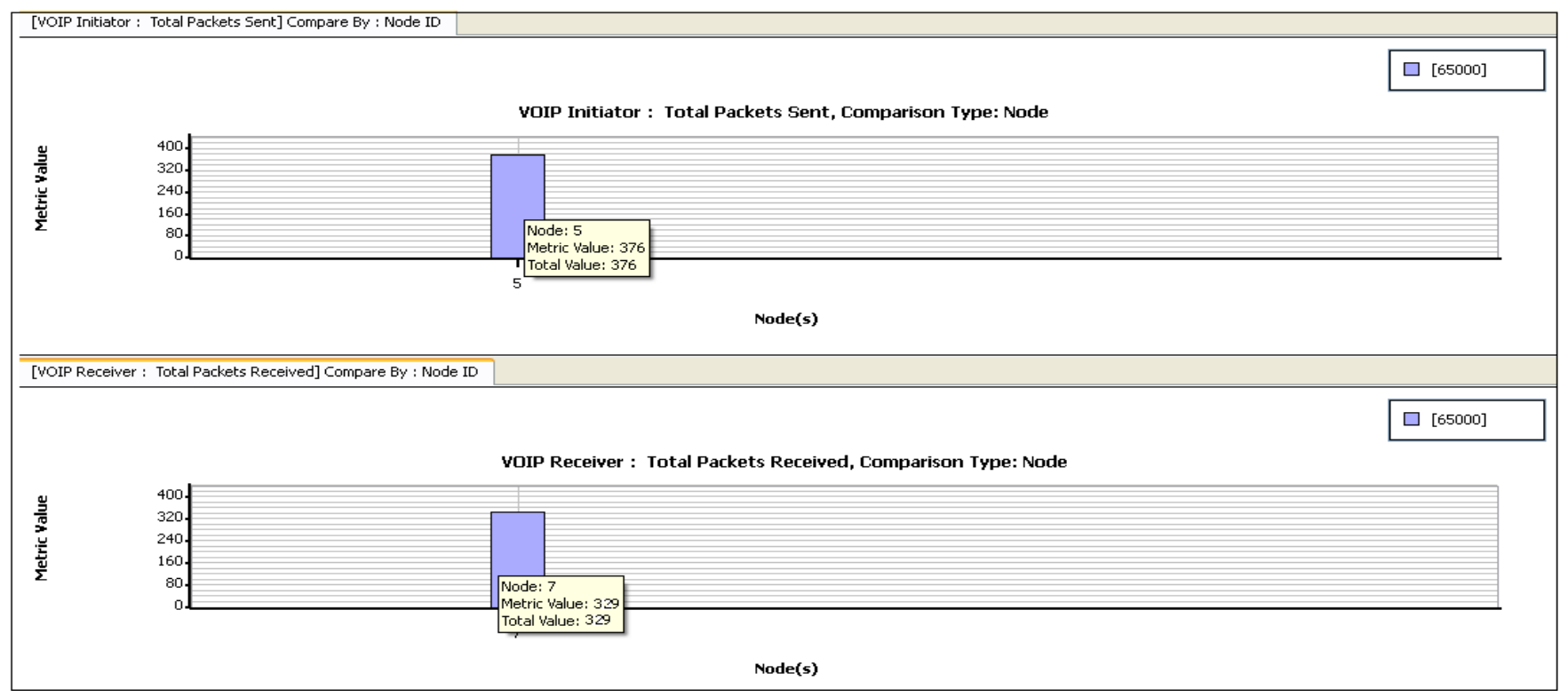

Figure: 5 simulated results for sending voice call from VOIP Client to VOIP Server using AODV routing protocol

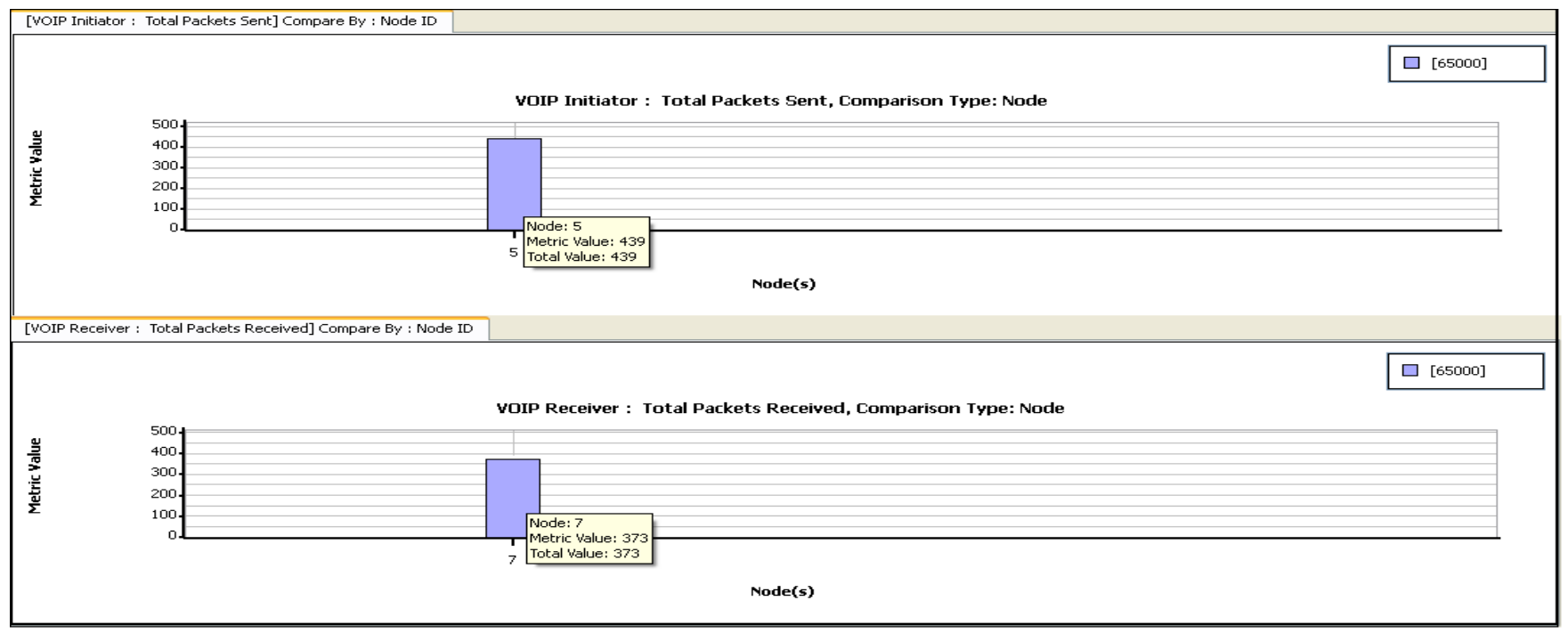

Figure: 6 simulated results for sending voice packet from VOIP Client to VOIP Server using Bellman ford routing protocol 


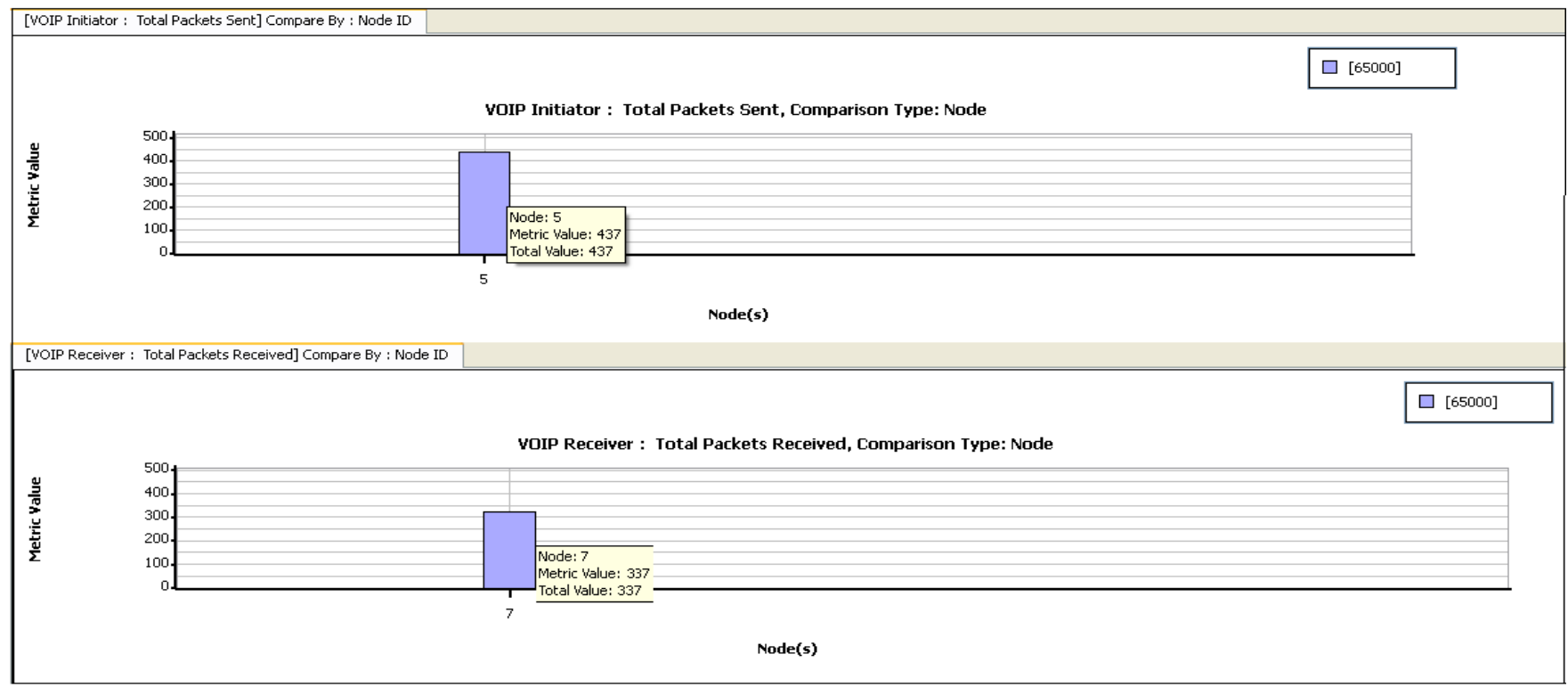

Figure: 7 simulated results for sending voice call packet from VOIP Client to VOIP Server using Fisheye routing protocol

\section{EXPERIMENTAL RESULTS}

Testing is conducted by the simulation mode for the wireless network. However simulation isn't always viewed as valuable or viable tool for comprehensive performance testing and evaluation, as there are vast differences in model fidelities and levels of abstraction between the actual system and the simulated model. In simulation, the model is often simplified or abstracted, which can mask the very phenomena (e.g., adaptive behavior) that are captured during testing on physical test beds. We tested our simulation scenario first with CBR link using different protocol. Using AODV protocol we tested our scenario figure 1 with CBR link by sending 9,400 packets shown in figure 2 and while reception of the traffic received by the CBR server is 8000 shown in the fig 2. Similarly Bellman ford and fisheye routing protocol had shown in the figure 3 and figure 4 respectively. Using these results we can predict that using CBR link AODV is efficient protocol compared to bellman ford and fisheye.

Second step we tested our simulation scenario with VoIP link using different protocol. Total number of voice packet sent from VoIP source to VoIP server shown in the table 1 using different protocol. Simulated result for sending VoIP client and VoIP server communication and total packet received by the VoIP server using different protocol showed in Figure 5, 6 and 7. After analysis we predicted that using VoIP traffic AODV, Bellman ford and Fisheye routing protocols works efficiently in our result.

\section{CONCLUSIONS AND FUTURE WORKS}

The paper focuses on the survey on VoIP linking over CBR linking, over three different implementations. Using the features of point to multicast mode simulation and calculations is concluded that VOIP emerges as the more efficient service-provider. The total packet sent and average packet lost rate, as can be observed for the results table 2, is considerably packet loss ration higher in CBR compared to VOIP traffic generator application.
As mentioned earlier, our research work has implemented scenarios in their ideal form. Future work will involve simulating scenarios that are closer to the real world models. Effects and consequences QoS factors, delays, packet loss probabilities and other environmental factors [13] will be considered in the future studies.

\section{ACKNOWLEDGMENTS}

The authors are thankful to the anonymous reviewers for their useful comments in improving the quality and presentation of this paper.

\section{REFERENCES}

[1] Vitor Bernardo, Bruno Sousa and Marilia Curado. 2009. VoIP over WiMAX: Quality of Experience Evaluation, In Proceedings of IEEE.

[2] Goode B. 2002. Voice over Internet Protocol (VoIP). Proceedings of IEEE, vol. 90, pp. 1495-517.

[3] M. Irfan Anis, M. Zamin Khan, Jahanzeb Inam and Rana Azmatullah. 2008. Comparison between the IEEE 802.11x Standards of VoIP Using the CBR and VBR Voice Schemes. In Proceedings of IEEE.

[4] Mo-Han Fong, Robert Novak, Sean McBeath and Roshni Srinivasan. 2008. Improved VoIP Capacity in Mobile WiMAX Systems Using Persistent Resource Allocation. IEEE Communications Magazine.

[5] Isha khirwar, Anjulata Yadav and Preeti Trivedi. 2010. Comparative Assessment of WiMAX Scheduler in Fixed and Mobile WiMAX Networks for VoIP using QualNet. In Proceedings of IEEE.

[6] Sheetal Jadhav, Haibo Zhang and Zhiyi Huang. 2011. Performance Evaluation of Quality of VoIP in WiMAX and UMTS. IEEE computer society.

[7] Dongmyoung Kim, Hua Cai, Minsoo Na, and Sunghyun Choi. 2008. Performance Measurement over Mobile WiMAX/IEEE 802.16e Network. In Proceedings of IEEE. 
[8] Zahian Ismail and Rosilah Hassan. 2010. Performance of AODV Routing Protocol in Mobile Ad Hoc Network. In Proceedings of IEEE.

[9] Marcus Scholler, Tarik Taleb, and Stefan Schmid. 2009. Neighborhoods as an Abstraction for Fish-Eye State Routing. In Proceedings of IEEE.

[10] Sandhya Kulkarni, H. J. Thontadharya, J.T. Devaraju, 2011. Performance Evaluation of VoIP in Mobile WiMAX: Simulation and Emulation studies. International Journal on Computer Science and Engineering (IJCSE).
[11] QualNet 4.5.1 documentation. Advanced wireless model library. http://www.scalablenetworks.com.

[12] S. Lirio Castellanos-Lopez, Mario E. Rivero-Angeles and Genaro Hernandez Valdez. 2012. Simplified Joint Call and Packet Level Teletraffic Analysis of CAC Strategies for VoIP Traffic in Wireless Networks.

[13] Joao Henriques, Vitor Bernardo, Paulo Simoes and Marilia Curado. 2012. VoIP performance over Mobile WiMAX: An Urban Deployment Analysis. 